IJMMS 29:1 (2002) 1-5

PII. S016117120200666X

http://ijmms.hindawi.com

(c) Hindawi Publishing Corp.

\title{
SUPER PARALLEL IMMERSIONS IN EUCLIDEAN SPACE
}

\author{
TAREK FATHY MERSAL and MOHAMED ESMAIL BASHER
}

Received 31 January 2001 and in revised form 20 May 2001

\begin{abstract}
Two submanifolds of Euclidean $n$-space $E^{n}$ are called super parallel if the affine normal spaces are homothetic at the corresponding points. Characterizations are given for the action of conformal transformation on super parallel mates. Our notion is generalized to super transnormal submanifolds and its relation with super self-parallel submanifolds and convex super self-parallel submanifolds.
\end{abstract}

2000 Mathematics Subject Classification: 53C20, 53C42.

1. Introduction. The notion of parallel and self-parallel smooth immersions $f, g$ of the smooth manifold $M$ into Euclidean $n$-space $E^{n}$ has been introduced by Farran and Robertson in [2]. Let $f: M \rightarrow E^{n}$ be a smooth immersion of an $m$-manifold $M$ into Euclidean $n$-space $E^{n}, n-m=k>0$ is the codimension of $M$. For any $x \in M$, the tangent map at $x$ is a linear map $T_{x} f: T_{x} M \rightarrow T_{y} E^{n}=E^{n}$ under the usual identification, $f(x)=y$. We denote by $L_{f, x}=T_{x} f\left(T_{x} M\right)$ the image of $T_{x} M$ as a linear subspace of $E^{n}$ whose dimension is the rank of $f$ at $x$, and by $L_{f, x}^{N}$ its orthogonal complement. The corresponding affine subspaces give the tangent spaces $\tau_{f}(x)=\{f(x)\}+L_{f, x}$ and the normal spaces $v_{f}(x)=\{f(x)\}+L_{f, x}^{N}$ to $f$ at $x$. The two immersions $f, g: M \rightarrow E^{n}$ are called parallel, denoted by $f \| g$, if for every $x \in M, v_{f}(x)=v_{g}(x)$. A diffeomorphism $J: M \rightarrow M$ of the domain of $f: M \rightarrow E^{n}$ is called a self-parallelism of $f$, if $f$ and $f \circ J$ are parallel. For more details see $[1,2,3,5]$.

\section{Super parallel immersions}

DEFinition 2.1. Two immersions $f, g: M \rightarrow E^{n}$ are called super parallel, denoted by $f \|_{s} g$ if for every $x \in M$, there exists homothety map $h_{\lambda}, \lambda \neq 0$,

$$
h_{\lambda}: v_{f}(x) \longrightarrow v_{g}(x) \text { given by } h_{\lambda}(\xi)=\lambda \xi .
$$

According to this definition, any two parallel immersions in a Euclidean space are super parallel immersions. The converse is not always true, for example, the conformal transformations of any two concentric spheres are super parallel submanifolds but not parallel submanifolds. We denote by $C=C^{\infty}\left(M, E^{n}\right)$ the set of all smooth maps $f: M \rightarrow E^{n}$. This is a real linear space. Let $I_{k} M$ denote the subset of $C$ consisting of all immersions of $M$ in $E^{n}$.

It is easy to see that the inverse of a homothety is also a homothety and the composite of a homothety of ratio $\lambda_{1}$ and a homothety of ratio $\lambda_{2}$ is a homothety of ratio $\lambda_{1} \lambda_{2}$. Then we have the following proposition.

Proposition 2.2. The relation $\|_{s}$ is an equivalence relation on $I_{k} M$. 
We will denote the super parallelism class of $f$ by $[f]_{s}$. Now we can form the affine hull $A(f)_{s}$ of $[f]_{s}$ for any $f$ in $C$. Thus $A(f)_{s}$ is the smallest affine subspace of $C$ that contains $[f]_{s}$. By the same procedure as in [2] we can prove that $[f]_{s}=I_{k} M \cap A(f)_{s}$. We generalize some properties that are well known in the parallel case (see $[3,5])$ to our consideration. Let $\bar{\nabla}$ denote the directional derivative in $E^{n}$. A normal field $\eta$ in the normal bundle of $f$ is called parallel if $\bar{\nabla}_{u} \eta$ is tangential, for all $u \in T_{x} M$ (see [5]).

LEMMA 2.3. The two immersions $f$ and $g$ of $M$ into $E^{n}$ are super parallel if and only if the vector field given by $\eta(x)=g(x)-\left(R_{g, \theta} \circ f\right)(x)$ is a parallel section of the normal bundle of $f$, where $\theta(x)$ is the directed angle between the vector $r(x)=g(x)-f(x)$ and the normal of $f$ at $x$, and $R_{g, \theta}$ is the rotation mapping about $g(x)$ through $\theta$.

Proof. Let $f, g$ be super parallel immersions in the Euclidean $n$-space $E^{n}$ and $\theta(x)$ is the angle between the vector $r(x)=g(x)-f(x)$ and the normal vector of $f$ at $x$. Then we can define a rotation map $R_{g, \theta}$ to $f(x)$ about $g(x)$ through directed angle $\theta$, such that $\eta(x)=g(x)-\left(R_{g, \theta} \circ f\right)(x)$ is a section of the normal bundle of $f$ at $x$ and $\left(R_{g, \theta} \circ f\right), g$ are parallel immersions in $E^{n}$. Hence by [5, Lemma 1] $\eta(x)=$ $g(x)-\left(R_{g, \theta} \circ f\right)(x)$ is a parallel section of the normal bundle of $f$.

Conversely, let $\eta(x)=g(x)-\left(R_{g, \theta} \circ f\right)(x)$ be a parallel section of the normal bundle of $f$, then from [5, Lemma 1] $\left(R_{g, \theta} \circ f\right), g$ are parallel immersions in $E^{n}$ and consequently $v_{g}(x)=v_{R_{g, \theta} \circ f}(x)$. Then if $\xi \in v_{g}(x)$, there exists $\zeta \in v_{R_{g, \theta} \circ f}(x)$ such that $\xi(x)=\zeta(x)$. Since $\zeta(x) \in \nu_{R_{g, \theta} \circ f}(x)$, hence there exists $\lambda \neq 0$ such that $\lambda \zeta(x) \in v_{f}(x)$. Thus we can define a homothety map $h_{\lambda}$,

$$
h_{\lambda}: v_{g}(x) \longrightarrow v_{f}(x), \quad \xi(x) \longmapsto \lambda \xi(x) .
$$

Then $f, g$ are super parallel immersions in the Euclidean $n$-space $E^{n}$.

Proposition 2.4. If the two immersions $f$ and $g$ of $M$ into $E^{n}$ are super parallel, then $\left\|g(x)-\left(R_{g, \theta} \circ f\right)(x)\right\|=$ constant.

Proof. Let $f, g$ be super parallel immersions in the Euclidean $n$-space $E^{n}$. Then from Lemma $2.3 \eta(x)=g(x)-\left(R_{g, \theta} \circ f\right)(x)$ is a parallel section in the normal bundle of $f$, that is, $\bar{\nabla}_{u} \eta$ is tangential, then

$$
\begin{aligned}
\bar{\nabla}_{u}\left\|g(x)-\left(R_{g, \theta} \circ f\right)(x)\right\|^{2} & =\bar{\nabla}_{u}\|\eta(x)\|^{2}=\bar{\nabla}_{u}\langle\eta(x), \eta(x)\rangle \\
& =2\left\langle\bar{\nabla}_{u} \eta(x), \eta(x)\right\rangle=0 .
\end{aligned}
$$

Hence $\left\|g(x)-\left(R_{g, \theta} \circ f\right)(x)\right\|=$ constant.

EXAMPLE 2.5. (1) Any two circles (hyperspheres) in $E^{n}$ are super parallel immersions.

(2) Let $M$ be an immersed submanifold in the Euclidean space $E^{n}$, and let $\tilde{M}$ be the reflection of $M$ with respect to the origin 0 , which is not inside $M$. Then $M$ and $\tilde{M}$ are super parallel immersed submanifolds in Euclidean space $E^{n}$.

(3) Let $\bar{M}$ be the translation of $M$ by a ratio $\mu$ in the Euclidean $n$-space $E^{n}$. Then $M$ and $\bar{M}$ are super parallel immersed submanifolds in Euclidean space $E^{n}$.

THEOREM 2.6. Let $f, g: M \rightarrow E^{n}$ be super parallel immersions in the Euclidean $n$-space $E^{n}$, then the immersed submanifolds $f(M)$ and $g(M)$ are diffeomorphic. 
Proof. Let $f, g: M \rightarrow E^{n}$ be two super parallel immersions in $E^{n}$, then from Lemma 2.3 there exists a parallel section $\eta(x)$ in the normal bundle of $f$, such that

$$
g(x)=\left(R_{g, \theta} \circ f\right)(x)+\eta(x) \quad \forall x \in M .
$$

Define a function

$$
J: f(M) \subset E^{n} \longrightarrow g(M) \subset E^{n}
$$

given by

$$
J(f(x))=\left(R_{g, \theta} \circ f\right)(x)+\eta(x)=g(x) .
$$

It is obvious that this function is differentiable. Also we can define a function

$$
J^{*}: g(M) \longrightarrow f(M)
$$

given by

$$
J^{*}(g(x))=R_{g, \theta}^{-1}(g(x)-\eta(x))=f(x),
$$

which is also differentiable and

$$
\begin{aligned}
J \circ J^{*}(g(x)) & =J\left(R_{g, \theta}^{-1}(g(x)-\eta(x))\right) \\
& =R_{g, \theta}\left(R_{g, \theta}^{-1}(g(x)-\eta(x))\right)+\eta(x) \\
& =g(x)-\eta(x)+\eta(x)=g(x), \\
J^{*} \circ J(f(x)) & =J^{*}\left(\left(R_{g, \theta} \circ f\right)(x)+\eta(x)\right) \\
& =R_{g, \theta}^{-1}\left(\left(R_{g, \theta} \circ f\right)(x)+\eta(x)-\eta(x)\right)=f(x) .
\end{aligned}
$$

Then $J \circ J^{*}=I$ and also $J^{*} \circ J=I$. Hence $J$ is a diffeomorphism, and $g(M)$ is diffeomorphic to $f(M)$.

COROLLARY 2.7. Let $f, g: M \rightarrow E^{n}$ be parallel immersions in the Euclidean $n$-space $E^{n}$, then the immersed submanifolds $f(M)$ and $g(M)$ are diffeomorphic.

Now we investigate the action of conformal transformation on super parallel mates in Euclidean space.

In the parallel case the conformal transformation $F$ preserved on parallel section but not preserved on parallel mates (see [4]). In our consideration we prove that the super parallel mates are preserved under conformal transformation.

THEOREM 2.8. The super parallel mates are preserved under conformal transformation of the ambient Euclidean space.

Proof. Let $f, g$ be super parallel immersions in $E^{n}$. Then we can define a homothety map $h_{\lambda}$ by $\lambda \neq 0$,

$$
\begin{gathered}
h_{\lambda}: v_{f}(x) \longrightarrow v_{g}(x) \text { given by } h_{\lambda}(\xi)=\lambda \xi, \\
v_{f}(x) \stackrel{h_{\lambda}}{\longrightarrow} v_{g}(x) \\
F \mid \begin{array}{l}
\mid F \\
v_{F \circ f}(x) \stackrel{h_{\lambda}^{*}}{\longrightarrow} v_{F \circ f}(x)
\end{array}
\end{gathered}
$$


The conformal map transfers the normal vector field on $f$ to a normal vector field on $F \circ f$ (see [6]). Then from the above diagram we can define a homothety map

$$
h_{\lambda}^{*}: v_{F \circ f}(x) \longrightarrow v_{F \circ g}(x), \quad h_{\lambda}^{*}\left(\xi^{*}\right)=\lambda \xi^{*},
$$

which means that $(F \circ f) \|_{s}(F \circ g)$ and this completes the proof.

3. Super self-parallel curves. Let $f: M \rightarrow E^{n}$ be an immersion. We say that $f$ is transnormal if, for every $x \in M, v_{f}(x)=v_{f}(y)$, whenever $f(y) \in v_{f}(x)$. Let $J: M \rightarrow M$ be a diffeomorphism of the domain of $f$, then $J$ is called self-parallelism of $M$ with respect to $f$ if $f$ and $f \circ J$ are parallel (see [1]).

DEFINITION 3.1. Let $f: M \rightarrow E^{n}$ be an immersion. We say that $f$ is super transnormal if, for every $x \in M$, there exists a unique point $y \in M$ such that $v_{f}(x)$ and $v_{f}(y)$ are homothetic, $h_{\lambda}: v_{f}(x) \rightarrow v_{f}(y), \lambda \neq 0$ whenever $f(y) \in h_{\lambda}\left(v_{f}(x)\right)$.

Homothety constant $\lambda=1$ implies the definition of transnormality given in [1]. Then transnormal immersions in Euclidean space $E^{n}$ are super transnormal. The general oval and ellipsoid are simple examples for super transnormal immersions which are not transnormal.

DEFinition 3.2. A diffeomorphism $J: M \rightarrow M$ of the domain of $f: M \rightarrow E^{n}$ is called a super self-parallelism of $f$, if $f$ and $f \circ J$ are super parallel.

Now we generalize some properties which exist in [1] to our consideration.

Proposition 3.3. Any convex super self-parallel embedding $f: S^{n} \rightarrow E^{n+1}$ is super transnormal.

Proof. Since $f\left(S^{n}\right)$ is convex super self-parallel, $L_{f, x}$ is the tangent plane of $f$ at $f(x)$, then there exists a unique point $f(y)$ such that $L_{f, y}= \pm L_{f, x}$. Then $L_{f, y}^{N}= \pm L_{f, x}^{N}$. Let $J$ be nontrivial super self-parallelism of $S^{n}$ with respect to $f$ and $x \in S^{n}$, then there is a homothety map $h_{\lambda}$ by $\lambda \neq 0$

$$
h_{\lambda}: v_{f}(x) \longrightarrow v_{f \circ J}(x) \text { given by } h_{\lambda}(\xi)=\lambda \xi \text {. }
$$

Hence we can define the homothety function $h_{\lambda}$ such that $h_{\lambda}: v_{f}(x) \rightarrow v_{f}(y)$, where $y=J(x)$ and $f(y) \in h_{\lambda}\left(v_{f}(x)\right)$. Then $f$ is super transnormal.

THEOREM 3.4. Let $f: S^{1} \rightarrow E^{2}$ be an embedding. Then $f$ is super transnormal if and only if it is super self-parallel.

Proof. Let $f: S^{1} \rightarrow E^{2}$ be super transnormal. Then if $\xi$ is normal at $x$, there exists a unique point $y \in S^{1}$ such that $h_{\lambda}(\xi)$ is normal at the point $y$, where $h_{\lambda}$ is a homothety map. So we can define a diffeomorphism $J: S^{1} \rightarrow S^{1}$ and consequently we can define a homothety map $h_{\lambda}: v_{f}(x) \rightarrow v_{f \circ J}(x)$ such that $h_{\lambda}(\xi)=\lambda \xi \in v_{f \circ J}(x)$. Then $f$ is super self-parallel curve. The proof of the converse by the same procedure as in [1].

THEOREM 3.5. Any convex embedding curve $f: S^{1} \rightarrow E^{2}$ is a super transnormal curve. 
Proof. Let $f$ be a closed convex plane curve and $p$ any point on $S^{1}, L_{f, p}$ is the tangent of $f$ at $p$. Then there exists $\bar{p} \in S^{1}$, such that $L_{f, p}= \pm L_{f, \bar{p}}$. Then $L_{f, p}^{N}= \pm L_{f, \bar{p}}^{N}$. Thus we can define a homothety map $h_{\lambda}$ by $\lambda \neq 0$

$$
h_{\lambda}: v_{f}(p) \longrightarrow v_{f}(\bar{p}) \text { given by } h_{\lambda}(\xi)=\lambda \xi
$$

Then $f$ is super transnormal.

COROLLARY 3.6. Any convex embedding curve $f: S^{1} \rightarrow E^{2}$ is a super self-parallel curve.

Proof. Let $f$ be a convex embedding, then from Theorem 3.5 it is super transnormal, and from Theorem 3.4 the curve which is super transnormal is super self-parallel curve.

\section{REFERENCES}

[1] F. J. C. de Carvalho and S. A. Robertson, Self-parallel curves, Math. Scand. 65 (1989), no. 1, 67-74.

[2] H. R. Farran and S. A. Robertson, Parallel immersions in Euclidean space, J. London Math. Soc. (2) 35 (1987), no. 3, 527-538.

[3] T. F. Mersal, Geometric and analytic considerations of the parallelism for submanifolds, Ph.D. thesis, Menoufia University, 1999.

[4] B. Wegner, Self-parallel and transnormal curves, Geom. Dedicata 38 (1991), no. 2, 175191.

[5] _ Some remarks on parallel immersions, Differential Geometry and Its Applications (Eger, 1989), Colloq. Math. Soc. Janos Bolyai, vol. 56, North-Holland, Amsterdam, 1992, pp. 707-717.

[6] _ Parallel immersions into spaces of constant curvature and conformal transformations, Gen. Math. 5 (1997), 401-407.

Tarek Fathy Mersal: Department of Mathematics, Faculty of Science, Menoufia UNIVERSITY, EGYPT

E-mail address: tmersa1@yahoo.com

Mohamed Esmail Basher: Department of Mathematics, Faculty of Education (Suez), SUEZ CANAL UNIVERSITY, EGYPT

E-mail address: m_e_basher@hotmai 1.com 


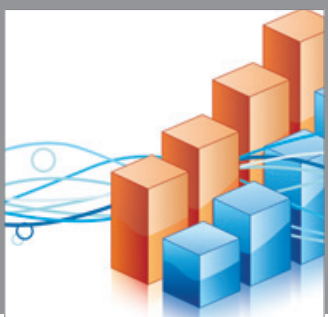

Advances in

Operations Research

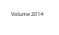

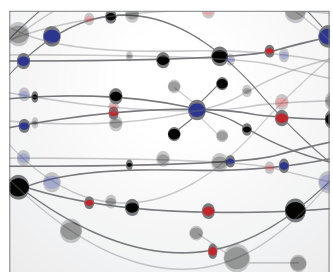

\section{The Scientific} World Journal
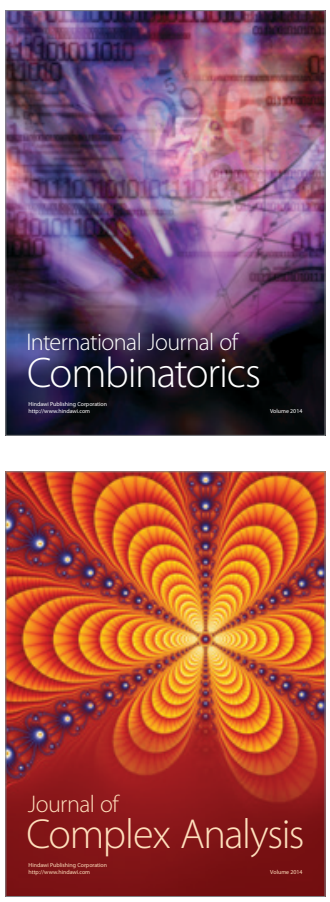

International Journal of

Mathematics and

Mathematical

Sciences
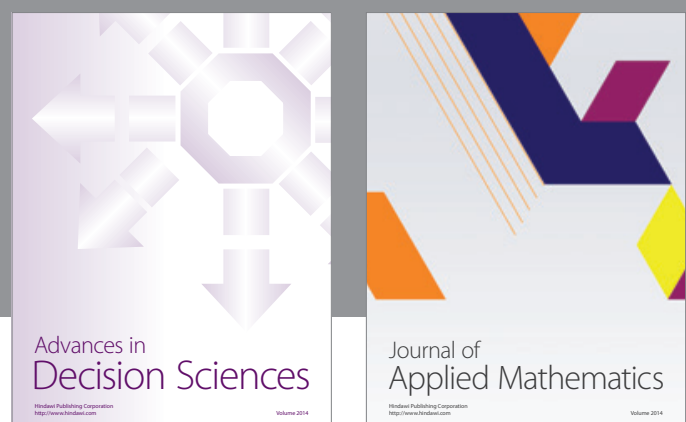

Journal of

Applied Mathematics
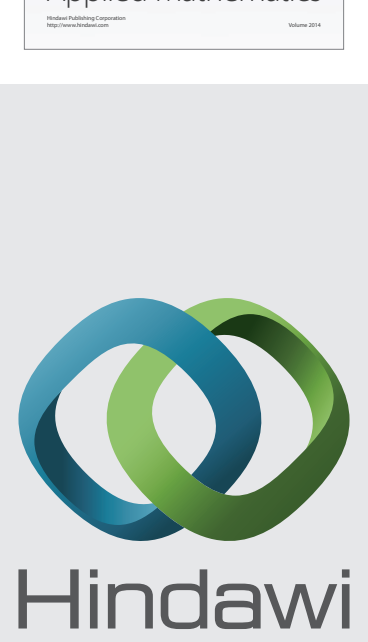

Submit your manuscripts at http://www.hindawi.com
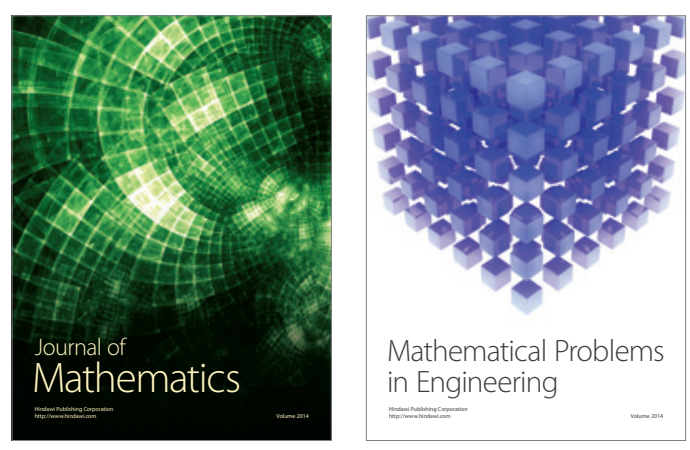

Mathematical Problems in Engineering
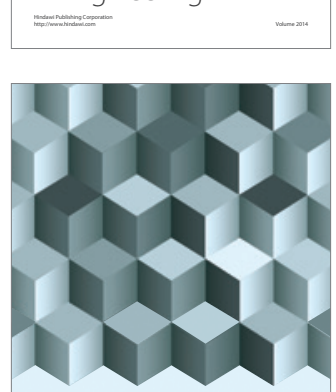

Journal of

Function Spaces
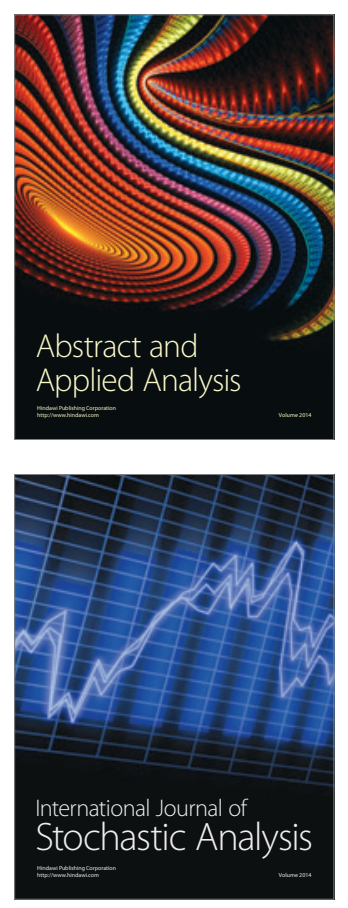

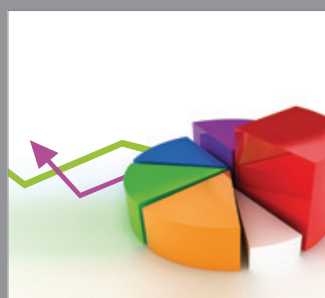

ournal of

Probability and Statistics

Promensencen
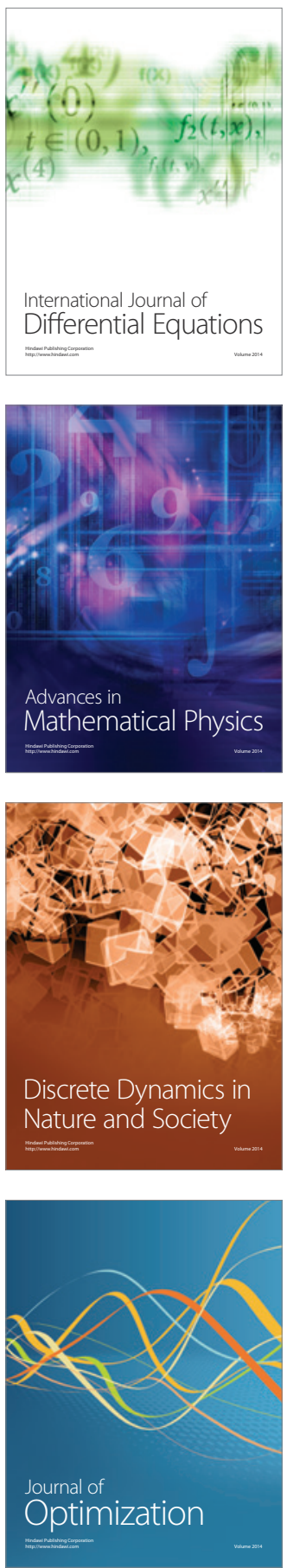